\title{
Comparación de la efectividad de ranibizumab intravítreo para el tratamiento del edema macular diabético en ojos vitrectomizados y no vitrectomizados
}

\author{
Yoshito Koyanagi Shigeo Yoshida Yoshiyuki Kobayashi Yuki Kubo \\ Muneo Yamaguchi Takahito Nakama Shintaro Nakao Yasuhiro Ikeda \\ Yuji Ohshima Tatsuro Ishibashi Kohhei Sonoda \\ Departamento de Oftalmología, Escuela de Graduados de Ciencias Médicas, Universidad de Kyushu, \\ Fukuoka, Japón
}

\section{Palabras clave}

Vitrectomía - Edema macular diabético · Anti-VEGF . Agudeza visual · Tomografía de coherencia óptica

\section{Resumen}

Objetivo: Comparar la efectividad de ranibizumab intravítreo (RIV) para el tratamiento del edema macular diabético (EMD) en ojos con y sin vitrectomía previa. Procedimientos: Evaluamos de manera prospectiva la mejor agudeza visual corregida (MAVC) y el grosor macular central (GMC) tras el tratamiento con RIV durante 6 meses. Resultados: No se observaron diferencias significativas en la MAVC o GMC inicial en ninguno de los dos grupos. En el grupo no vitrectomizado $(n=15)$, los cambios medios en la MAVC y GMC hasta el sexto mes de tratamiento con respecto al valor inicial resultaron significativos $(p<0,01)$. En el grupo vitrectomizado $(n=10)$, se observó una mejora más lenta, y la mejora media en la MAVC no resultó significativa $(p=0,5)$, aunque la media en la disminución del GMC sí que lo fue $(p<0,05)$. No se observaron diferencias significativas en los cambios medios en la MAVC y el GMC entre ambos grupos a los 6 meses del tratamiento. Conclusiones: La diferencia en la efectividad de RIV entre ambos grupos no resultó significativa. Ranibizumab intravítreo puede ser una opción de tratamiento incluso en pacientes vitrectomizados con EMD.

(c) 2017 S. Karger AG, Basel

\section{Introducción}

El edema macular diabético (EMD) representa la causa más frecuente de reducción de la visión en pacientes con retinopatía diabética [1]. El factor de crecimiento del endotelio vascular (VEGF, por sus siglas en inglés) es un importante mediador de la permeabilidad vascular anormal en pacientes con EMD [2,3], y varios ensayos clínicos han confirmado que las inyecciones intravítreas de anticuerpos anti-VEGF consiguieron mejores resultados visuales que el uso de fotocoagulación macular con láser, que desde los años 80 ha sido el tratamiento de referencia para el EMD [4-14].

The Spanish version should be cited as DOI: 10.1159/000477513. The original version is in English and should be cited as DOI: 10.1159/000446992.

\section{KARGER}

(C) 2017 S. Karger AG, Basel
Shigeo Yoshida, MD, PhD

Departamento de Oftalmología, Escuela de Graduados de Ciencias Médicas Universidad de Kyushu, 3-1-1 Maidashi, Higashi-ku

Fukuoka 812-8582 (Japón)

E-Mail yosida@eye.med.kyushu-u.ac.jp 
Existen datos limitados y contradictorios sobre la efectividad del tratamiento anti-VEGF en ojos que se han sometido a vitrectomía. Estudios con animales indicaron que la semivida de los fármacos anti-VEGF inyectados en el vítreo de los ojos sometidos a vitrectomía era menor que la observada en ojos no vitrectomizados $[15,16]$. Además, se ha demostrado que la concentración del VEGF en el cuerpo vítreo disminuye tras la vitrectomía $[17,18]$. Solamente un número reducido de estudios han examinado la efectividad del tratamiento anti-VEGF en ojos humanos vitrectomizados, y los resultados obtenidos resultan incongruentes. Dos estudios de corta duración demostraron que bevacizumab tenía muy poco o ningún efecto en ojos vitrectomizados $[19,20]$. Un estudio reciente de DRCR.net mostró que la vitrectomía previa no afectó a la utilidad de ranibizumab intravítreo (RIV) [21].

Por ello, el objetivo del presente estudio comprendía comparar la efectividad de RIV para el tratamiento del EMD en ojos con y sin vitrectomía previa.

\section{Materiales y métodos}

Nuestro estudio fue aprobado por el Comité de Ética Institucional del Hospital Universitario de Kyushu (Fukuoka, Japón) y se llevó a cabo de acuerdo con los principios de la Declaración de Helsinki sobre investigación biomédica en seres humanos. Todos los pacientes dieron su consentimiento informado antes de su inclusión en el estudio.

Se estudió a todos los pacientes que padecían EMD y que recibieron inyecciones programadas de RIV. Todos los pacientes fueron examinados en el Hospital Universitario de Kyushu entre febrero de 2014 y abril de 2015 y comparamos los datos recogidos antes de administrar RIV con los datos obtenidos 6 meses después de administrar RIV.

En este estudio prospectivo se estudiaron 25 ojos de 20 pacientes, y todos ellos tenían EMD persistente. De estos 25 ojos, 10 que habían sido sometidos a vitrectomía para el tratamiento del EMD al menos 3 meses antes de la inyección de RIV fueron ubicados en el grupo vitrectomizado, y 15 ojos sin vitrectomía previa conformaron el grupo no vitrectomizado. Los criterios de inclusión comprendieron la mejor agudeza visual corregida (MAVC) decimal $<0,7 \mathrm{y} / \mathrm{o}$ el grosor macular central $(\mathrm{GMC})>350 \mu \mathrm{m}$ medido mediante tomografía de coherencia óptica (OCT, por sus siglas en inglés). Los ojos excluidos poseían alteraciones que podían impedir una mejora de la MAVC, inflamación intraocular o infección en uno de los dos ojos, glaucoma no controlado en alguno de los ojos o habían sido sometidos a panfotocoagulación con láser en los 6 meses previos o fotocoagulación local/en rejilla en los 3 meses previos al comienzo de este estudio. También se excluyó a los pacientes que se hubieran sometido previamente a tratamiento con un fármaco antiangiogénico en el ojo estudiado en los 3 meses previos a las inyecciones de RIV, o a los que se les hubiera practicado una vitrectomía, una extracción de cataratas o hubieran recibido inyecciones de esteroides durante el transcurso del presente estudio.
La dosis intravítrea de ranibizumab fue de $0,5 \mathrm{mg} / 0,05 \mathrm{ml}$. Inicialmente, los pacientes recibieron 3 inyecciones mensuales consecutivas de ranibizumab y después, en los casos en que fuera necesario, hasta que se confirmara una mejora del edema macular central mediante una OCT o hasta que se estabilizara la agudeza visual. Se definió como mejora anatómica una disminución de $>10$ $\mu \mathrm{m}$ del GMC, teniendo en cuenta los sesgos de mediciones entre exploraciones.

Se realizaron análisis estadísticos con un paquete estadístico comercial (IMP, versión 11.0; SAS Institute, Cary, N.C., EE. UU.). La MAVC se midió con optotipos decimales japoneses estándar y se convirtió a unidades del logaritmo en el ángulo mínimo de resolución (logMAR). Inicialmente, se utilizó el test de Shapiro-Wilk para determinar si los datos se habían distribuido en condiciones normales. La significación de las diferencias en la edad, la HbA1c y el número de inyecciones entre el grupo vitrectomizado y el grupo no vitrectomizado se determinó mediante pruebas de la $t$ de Student. Las pruebas del orden de la suma de Wilcoxon se utilizaron para determinar si existía una diferencia significativa en la MAVC y el GMC iniciales y el intervalo desde el primer diagnóstico de EMD hasta el momento de recibir RIV entre el grupo vitrectomizado y el grupo no vitrectomizado. Además, se utilizó la prueba exacta de Fisher para determinar si la distribución de sexos resultaba significativamente distinta entre ambos grupos. Se evaluaron los cambios medios en la MAVC y la GMC hasta el sexto mes con respecto al valor inicial mediante prueba de la $t$ para datos emparejados. La significación de las diferencias en los cambios medios en la MAVC y el GMC hasta el sexto mes con respecto al valor inicial entre los dos grupos se comparó mediante pruebas de la $t$ de Student. La correlación entre el momento a partir del primer diagnóstico de EMC y los cambios en la MAVC y el GMC se determinó utilizando el coeficiente de correlación de Spearman. Los valores ausentes se asignaron mediante el método de traslación del último dato. Las diferencias se consideraron estadísticamente significativas con $\mathrm{p}<0,05$.

\section{Resultados}

Los datos sociodemográficos y las características iniciales de los pacientes se muestran en la tabla 1 . Antes de iniciar el estudio, los datos referentes a la edad, la distribución de sexos, el control glucémico, la MAVC y el GMC del grupo vitrectomizado no resultaron significativamente distintos de los del grupo no vitrectomizado. No obstante, el intervalo entre el primer diagnóstico de EMD y las inyecciones de RIV fue más largo en el grupo vitrectomizado que en el grupo no vitrectomizado.

A todos los pacientes se les realizó seguimiento durante 6 meses. A los 6 meses de tratamiento, el número medio de inyecciones de RIV en ojos no vitrectomizados no resultó significativamente distinto al de ojos vitrectomizados (tabla 2).

En el grupo no vitrectomizado, la MAVC media $\pm \mathrm{DE}$ fue $0,460 \pm 0,288$ unidades $\log M A R$ antes de comenzar el estudio y $0,315 \pm 0,364$ unidades $\log$ MAR a los seis me-
Ophthalmologica 2017;238(suppl 1):21-27 DOI: $10.1159 / 000477513$
Koyanagi et al. 
Tabla 1. Características iniciales

\begin{tabular}{|c|c|c|c|}
\hline Características & $\begin{array}{l}\text { No vitrectomizados } \\
(\mathrm{n}=15)\end{array}$ & $\begin{array}{l}\text { Vitrectomizados } \\
(\mathrm{n}=10)\end{array}$ & $\mathrm{p}$ \\
\hline \multicolumn{4}{|l|}{ Edad, años } \\
\hline Media $\pm \mathrm{DE}$ & $69,7 \pm 10,0$ & $66,0 \pm 6,3$ & \multirow[t]{2}{*}{$0,306^{\mathrm{b}}$} \\
\hline Intervalo & $50-83$ & $53-72$ & \\
\hline Hombres/mujeres, $\mathrm{n}^{\mathrm{a}}$ & $6 / 7$ & $3 / 5$ & $1.000^{\mathrm{c}}$ \\
\hline \multicolumn{4}{|l|}{ Glucohemoglobina, \% } \\
\hline Media $\pm \mathrm{DE}$ & $7,1 \pm 0,9$ & $7,0 \pm 1,0$ & \multirow[t]{2}{*}{$0,746^{\mathrm{b}}$} \\
\hline Media (percentil $25^{\circ}, 7^{\circ}$ ) & $6,9(6,5,7,8)$ & $6,8(6,4,7,3)$ & \\
\hline \multicolumn{4}{|c|}{ Tiempo transcurrido desde el primer diagnóstico de EMD, meses } \\
\hline Media \pm DE & $16,2 \pm 24,4$ & $38,3 \pm 16,7$ & \multirow[t]{2}{*}{$0,002^{\mathrm{d}}$} \\
\hline Intervalo & $0-82$ & $28-84$ & \\
\hline \multicolumn{4}{|l|}{ MAVC logMAR } \\
\hline Media $\pm \mathrm{DE}$ & $0,46 \pm 0,29$ & $0,51 \pm 0,37$ & \multirow[t]{2}{*}{$0,867^{\mathrm{d}}$} \\
\hline Media (percentil 25, $75^{\circ}$ ) & $0,40(0,22,0,70)$ & $0,46(0,14,0,87)$ & \\
\hline \multicolumn{4}{|l|}{$\mathrm{GMC}, \mu \mathrm{m}$} \\
\hline Media \pm DE & $485 \pm 133$ & $635 \pm 307$ & \multirow[t]{2}{*}{$0,285^{\mathrm{d}}$} \\
\hline Media (percentil $25^{\circ}, 75^{\circ}$ ) & $467(388,627)$ & $568(409,762)$ & \\
\hline \multicolumn{4}{|l|}{ Características oculares } \\
\hline PFC previa & $8(53 \%)$ & $9(90 \%)$ & \\
\hline STAT previa & $7(47 \%)$ & $10(100 \%)$ & \\
\hline Tratamiento previo con anti-VEGF para EMD & $2(13 \%)$ & $5(50 \%)$ & \\
\hline \multicolumn{4}{|l|}{ Estado de lente } \\
\hline Fáquico & $6(40 \%)$ & 0 & \\
\hline Pseudofáquico & $9(60 \%)$ & $10(100 \%)$ & \\
\hline \multicolumn{4}{|l|}{ Tiempo transcurrido desde la vitrectomía } \\
\hline$<1$ año & & $2(20 \%)$ & \\
\hline $1 \mathrm{a}<2$ años & & $2(20 \%)$ & \\
\hline$\geq 2$ años & & $6(60 \%)$ & \\
\hline Media \pm DE, meses & & $25,98 \pm 14,0$ & \\
\hline Media (intervalo), meses & & $26,7(9,3-55,0)$ & \\
\hline
\end{tabular}

ses. En el grupo vitrectomizado, la MAVC media $\pm \mathrm{DE}$ fue $0,509 \pm 0,370$ unidades logMAR antes de comenzar el estudio y $0,467 \pm 0,370$ unidades $\log$ MAR a los seis meses. El cambio medio en la MAVC hasta el sexto mes con respecto al valor inicial resultó significativo en el grupo no vitrectomizado $(p=0,007)$, pero no lo fue en el grupo vitrectomizado ( $\mathrm{p}=0,521)$. 1, 3 y 6 meses después de administrar RIV, el grupo de ojos no vitrectomizados tenía una mejora media de $-0,062,-0,144$ y $-0,145$ unidades $\log$ MAR, respectivamente, mientras que el grupo de ojos con vitrectomía previa tenía una mejora media de $-0,029$, $-0,089$ y $-0,042$ unidades $\operatorname{logMAR}$, respectivamente (fig. 1). En la visita a los 6 meses, la mejora de la agudeza visual en el grupo no vitrectomizado fue $-0,103$ unidades $\operatorname{logMAR}$ mejor que en el grupo vitrectomizado, aunque

Ranibizumab para el tratamiento del EMD después de una vitrectomía la diferencia no resultó significativa entre ambos grupos ( $\mathrm{p}=0,189$; tabla 2). En la visita a los 6 meses, el 73\% de los ojos del grupo no vitrectomizado tenía más de 0,1 unidades logMAR de mejora en la MAVC, y un $40 \%$ de los ojos del grupo vitrectomizado tenía más de 0,1 unidades logMAR de mejora en la MAVC (tabla 2). Además, el $80 \%(12 / 15)$ de los ojos en el grupo no vitrectomizado y un 60\% (6/10) de los ojos en el grupo vitrectomizado mostraba mejoras a los 6 meses (fig. 2).

En el grupo no vitrectomizado, el GMC medio \pm DE fue $485 \pm 133 \mu \mathrm{m}$ antes de comenzar el estudio y $334 \pm$ $110 \mu \mathrm{m}$ a los 6 meses. En el grupo no vitrectomizado, el GMC medio \pm DE fue $635 \pm 307 \mu \mathrm{m}$ antes de comenzar el estudio y $450 \pm 210 \mu \mathrm{m}$ a los 6 meses. Se observó una mejora significativa del GMC en ambos grupos a los 6

Ophthalmologica 2017;238(suppl 1):21-27 23 


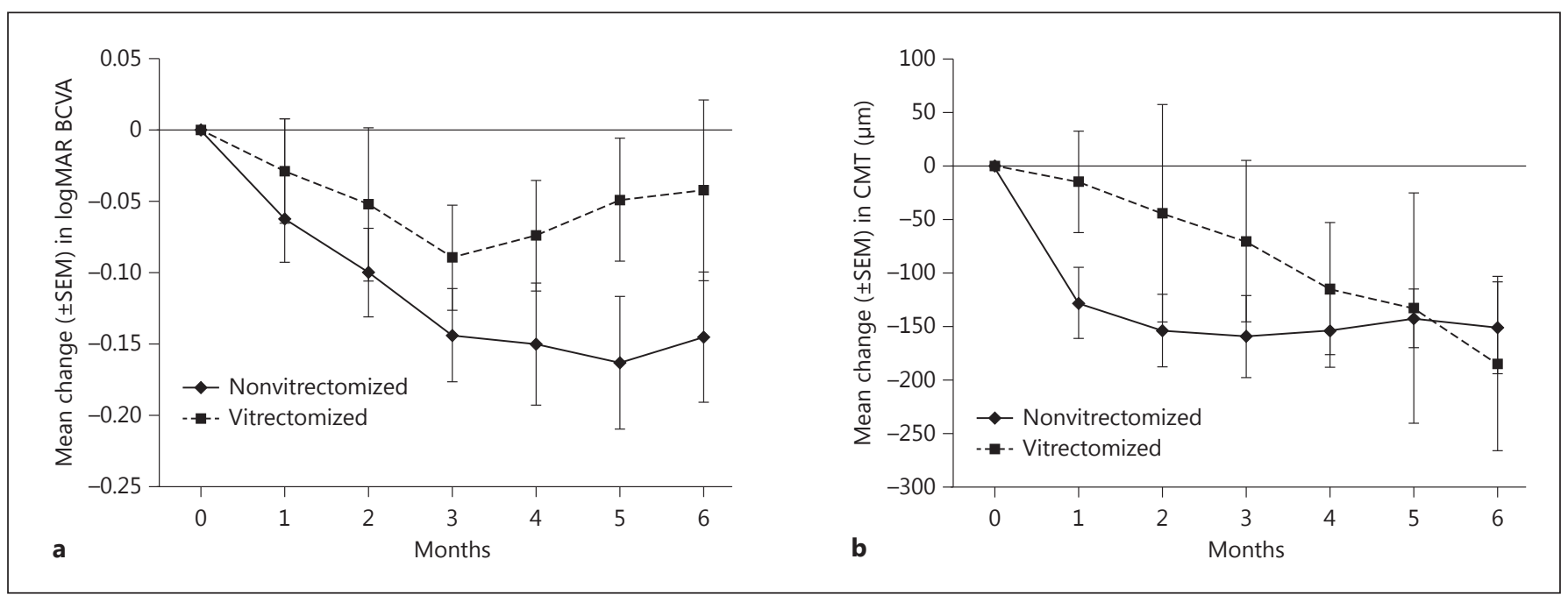

Fig. 1. Efecto de las inyecciones de RIV en la MAVC y el GMC en ojos previamente vitrectomizados y no vitrectomizados. a Cambio medio en la MAVC en unidades logMAR hasta el sexto mes con respecto al valor inicial en ojos previamente vitrectomizados y no vitrectomizados. b Cambio medio en el GMC en unidades logMAR hasta el sexto mes con respecto al valor inicial en ojos previamente vitrectomizados y no vitrectomizados. SEM = error estándar de las medias.

Tabla 2. Resultados de MAVC y GMC al sexto mes

\begin{tabular}{|c|c|c|c|}
\hline & $\begin{array}{l}\text { No vitrectomizado } \\
(\mathrm{n}=15)\end{array}$ & $\begin{array}{l}\text { Vitrectomizado } \\
(\mathrm{n}=15)\end{array}$ & $\mathrm{p}^{\mathrm{a}}$ \\
\hline \multicolumn{4}{|c|}{ Cambio en MAVC logMAR hasta el sexto mes con respecto al valor inicial } \\
\hline Media $\pm \mathrm{DE}$ & $-0,145 \pm 0,177$ & $-0,042 \pm 0,200$ & 0,189 \\
\hline Media (intervalo) & $-0,176(-0,477$ a 0,301$)$ & $-0,097(-0,301$ a 0,352$)$ & \\
\hline IC del $95 \%$ para la media & $-0,243 \mathrm{a}-0,047$ & $-0,185$ a 0,101 & \\
\hline \multicolumn{4}{|c|}{ Cambio de GMC hasta el sexto mes con respecto al valor inicial } \\
\hline Media \pm DE & $-151,5 \pm 165,9$ & $-184,6 \pm 257,9$ & 0,699 \\
\hline Media (intervalo) & $-123(-464$ a 130$)$ & $-125(-658$ a 151$)$ & \\
\hline IC del 95\% para la media & $-243,4$ a $-59,6$ & $-369,1$ a $-0,1$ & \\
\hline \multicolumn{4}{|c|}{ Cambio categórico en MAVC con respecto al valor inicial, n (\%) } \\
\hline Mejora $\log M A R \geq 0,15$ & $8(53)$ & $4(40)$ & \\
\hline Mejora $\log M A R \geq 0,1$ & $11(73)$ & $4(40)$ & \\
\hline Empeoramiento con respecto al valor inicial & $2(13)$ & $3(30)$ & \\
\hline Inyecciones de RIV, media $\pm \mathrm{DE}, \mathrm{n}$ & $4,5 \pm 1,2$ & $4,9 \pm 1,3$ & 0,484 \\
\hline
\end{tabular}

meses (fig. 1, $\mathrm{p}=0,003$ en el grupo no vitrectomizado, $\mathrm{y}$ $\mathrm{p}=0,0499$ en el grupo vitrectomizado). La mejora media del GMC fue -128, -159 y - $152 \mu \mathrm{m}$ en el grupo no vitrectomizado en las visitas del primer, tercer y sexto mes, respectivamente, frente a $-15,-71$ y $-185 \mu \mathrm{m}$, respectivamente, en el grupo vitrectomizado.

El cambio medio en el GMC hasta el sexto mes con respecto al valor inicial no resultó significativamente distinto entre el grupo vitrectomizado y el no vitrectomiza- do $(\mathrm{p}=0,699$; tabla 2$)$. Nuestros resultados mostraron que el $87 \%$ (13/15) de los ojos en el grupo no vitrectomizado y el 80\% (8/10) de los ojos en el grupo vitrectomizado mostraba mejoras anatómicas a los 6 meses (fig. 2).

Evaluamos también las correlaciones entre el momento en el que se realizó el primer diagnóstico de EMC y los cambios en la MAVC y el GMC porque existía una diferencia significativa en el tiempo entre los dos grupos. No obstante, la correlación entre el tiempo y los cambios en 


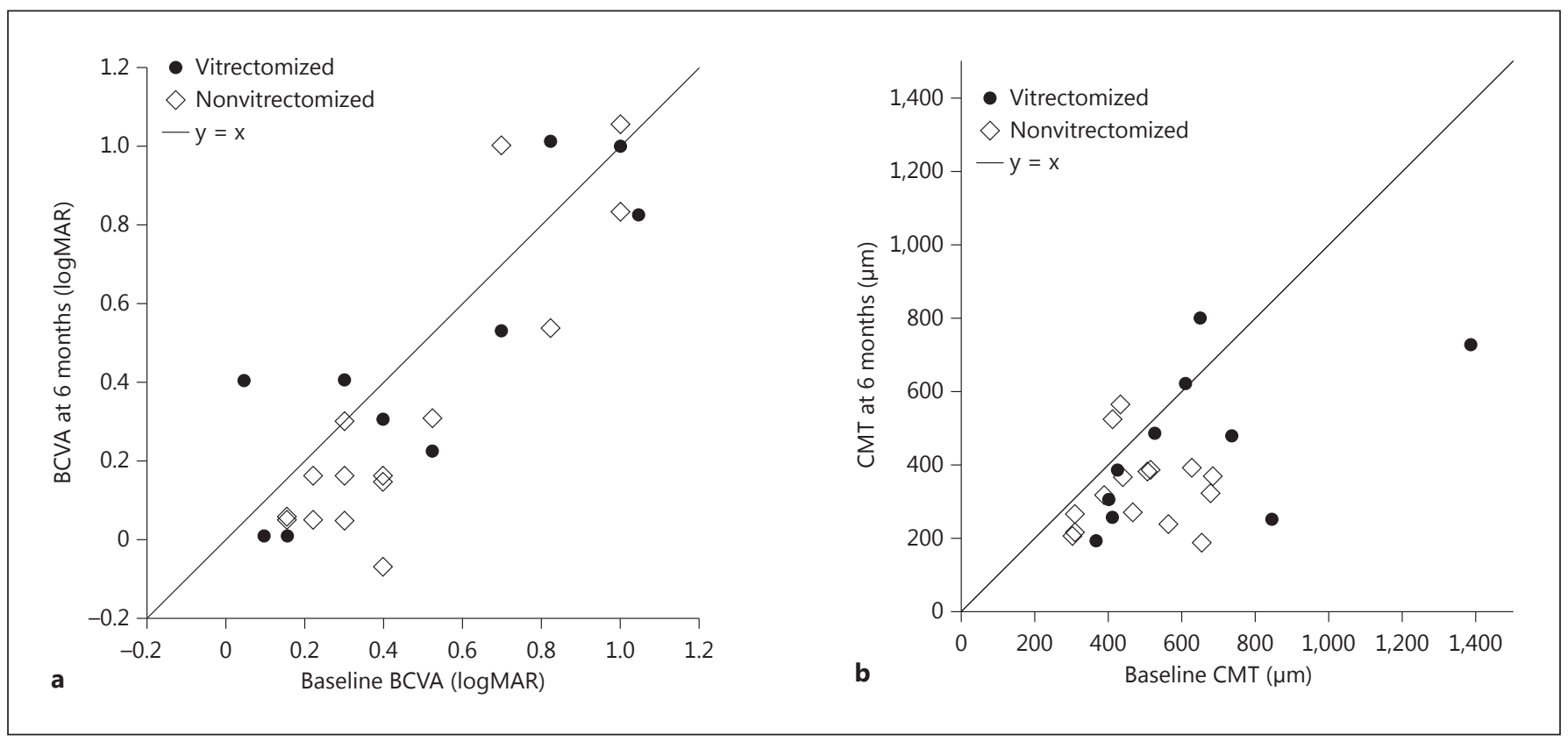

Fig. 2. Efecto de las inyecciones de RIV en la MAVC y el GMC en ojos previamente vitrectomizados y no vitrectomizados. a Diagrama de dispersión que compara la MAVC antes de comenzar el estudio y seis meses después en ojos previamente vitrectomizados y no vitrectomizados. b Diagrama de dispersión que compara el GMC antes de comenzar el estudio y seis meses después en ojos previamente vitrectomizados y no vitrectomizados.

la MAVC $(\mathrm{r}=0,331, \mathrm{p}=0,106)$ y el GMC $(\mathrm{r}=-0,116$, $\mathrm{p}=0,581)$ no resultó significativa. No se detectaron complicaciones clínicamente significativas en ninguno de los dos tipos de ojo.

\section{Discusión}

Nuestros resultados mostraron que las diferencias en la MAVC y el GMC entre el grupo vitrectomizado y el grupo no vitrectomizado no resultaron significativas a los 6 meses del tratamiento tras la primera inyección de RIV. No obstante, en el grupo vitrectomizado las mejoras fueron más lentas y la mejora media en la MAVC no fue significativa. Los ojos en ambos grupos mostraron reducciones significativas del GMC. Estos resultados indican que el uso de RIV todavía puede considerarse una opción de tratamiento incluso en pacientes con EMD vitrectomizados, aunque las mejoras se producen más lentas.

A los 6 meses del tratamiento, el cambio medio en la MAVC en el grupo no vitrectomizado fue $-0,15$ unidades $\log M A R$, que es equivalente a una mejora de 7 a 8 letras en la tabla del ETDRS. Este hallazgo concuerda con los resultados del estudio RESTORE (6-7 letras) y el estudio
RISE (9-10 letras) [6, 11]. La mejora en la MAVC en el grupo de ojos vitrectomizados no resultó significativa, aunque la mejora en el GMC en este mismo grupo fue equivalente a la del grupo de ojos no vitrectomizados. Señalamos que la ausencia de una mejora significativa en la MAVC en el grupo de ojos vitrectomizados quizá se deba a un daño grave de los fotorreceptores provocado por un intervalo más largo entre el diagnóstico inicial del EMD y la primera inyección de RIV. Sin embargo, la correlación existente entre el intervalo y la mejora en la MAVC no resultó significativa. Hubo 2 ojos vitrectomizados cuya MAVC empeoró a pesar de sus mejoras anatómicas. La zona elipsoidal detectada mediante OCT ya se encontraba afectada antes de las inyecciones de RIV, y esta situación permaneció invariable 6 meses después de la primera administración de RIV. Señalamos que esta fue la razón por la que las mejoras visuales de estos pacientes fueron pequeñas a pesar de sus mejoras anatómicas. Además, la mejora anatómica se debió principalmente a la mejora del edema macular central, y el efecto de adelgazamiento de la retina sensorial por las inyecciones de RIV fue relativamente escaso. Si la zona elipsoidal se encuentra afectada o se encuentra ausente antes de administrar la inyección de RIV, existe la posibilidad de que
Ranibizumab para el tratamiento del EMD después de una vitrectomía
Ophthalmologica 2017;238(suppl 1):21-27 DOI: $10.1159 / 000477513$ 
no se consigan mejoras visuales, de modo que se debe considerar con atención el tratamiento con RIV.

Sugerimos que la lenta recuperación del GMC en el grupo vitrectomizado podría deberse a la diferencia en el aclaramiento del fármaco [15] y los cambios en el perfil de expresión de citocinas tras la vitrectomía. Se ha demostrado que la concentración del VEGF es inferior [17] y que la concentración de citocinas inflamatorias, tales como JL-6 y MCP-1 es superior aproximadamente unos 7 meses tras una vitrectomía satisfactoria [18]. Consideramos que dichas citocinas inflamatorias podrían ocasionar un EMD prolongado tras la vitrectomía. Además, estudios anteriores han demostrado que las citocinas inflamatorias, tales como la IL-6, estimulan la expresión del VEGF en varios tipos de células distintas [22, 23]. Otros estudios han demostrado que la activación de la vía de transducción de señales de la IL-6 provocó la fosforilación de STAT3, lo que conllevó un aumento de la producción del VEGF $[24,25]$. A partir de estos resultados, apuntamos que el nivel reducido de concentración intravítrea de VEGF tras vitrectomía [18] se restablece de forma gradual mediante la estimulación de estas citocinas inflamatorias sobreexpresadas. Así, esto debería conllevar una recuperación más lenta del GMC y una prolongación del EMD por el fármaco anti-VEGF en los ojos vitrectomizados.

Informamos anteriormente que el nivel intravítreo de MCP-1 era significativamente superior en ojos vitrectomizados con EMD en comparación con el de VEGF [18]. Por ello, indicamos que el uso de MCP-1 podría estar estrechamente relacionado con un EMD prolongado tras una vitrectomía en comparación con el VEGF. No obstante, a juzgar por las lentas mejoras en los ojos vitrectomizados tras recibir tratamiento anti-VEGF, el factor de crecimiento del endotelio vascular se encuentra sin duda ligado a un EMD prolongado tras vitrectomía, aunque el efecto podría resultar relativamente pequeño en comparación con el EMD en ojos no vitrectomizados. Además, un estudio anterior [26] demostró que la MCP-1 era inducida por el VEGF y participaba en la angiogénesis y la permeabilidad vascular inducida por el VEGF. De este modo, el efecto de la MCP-1 podría verse suprimido por el tratamiento anti-VEGF de manera indirecta. Además, los tratamientos con corticoesteroides que suprimen las citocinas inflamatorias, incluyendo la IL-6 y la MCP-1 no pueden continuar administrándose en casos con complicaciones, tales como progresión de cataratas o elevación de la presión intraocular. En conjunto, los fármacos anti-VEGF deberían considerarse una de las principales opciones para el tratamiento del EMD en ojos vitrectomizados.
En la clínica, los tratamientos anti-VEGF no siempre se consideran como opción para EMD persistente tras vitrectomía. No obstante, nuestros resultados sugieren que, aunque las mejoras aparecieran con mayor lentitud en los ojos vitrectomizados, finalmente no existiría una diferencia significativa en el efecto de RIV entre ojos vitrectomizados y no vitrectomizados. Estos resultados son concordantes con un informe anterior [21].

La solidez de nuestro estudio se fundamenta en los datos que recogimos de forma prospectiva y en la ausencia de diferencias significativas en los niveles iniciales de MAVC y GMC entre ambos grupos. Esta información se contrasta con un estudio anterior [21]. Se sabe que los niveles iniciales de MAVC y GMC están asociados a los resultados visuales y de OCT tras un tratamiento antiVEGF [27]. Por ello, redujimos los efectos de estos posibles factores de confusión. Sin embargo, el EMD persistente en ojos vitrectomizados podría resultar más refractario que en ojos no vitrectomizados dado que muchos casos de EMD pueden resolverse o mejorarse tras someterse a una vitrectomía. Según lo anterior, los resultados de este estudio deberían interpretarse con cautela. Además, debido al reducido número de ojos con vitrectomía y a la ausencia de aleatorización por el estado de la vitrectomía en condiciones basales, se requieren estudios adicionales que proporcionen una mejor comprensión de la fisiopatología del EMD después de una vitrectomía.

En conclusión, la diferencia en la efectividad de RIV entre los grupos de ojos vitrectomizados y no vitrectomizados no resultó significativa. Nuestros resultados indican que la administración de RIV puede resultar una opción de tratamiento incluso en pacientes con EMD vitrectomizados, aunque las mejoras son más lentas.

\section{Agradecimientos}

Este trabajo fue financiado en parte por ISPS KAKENHI, subvenciones 26293374, 26670757 y 15 H04995 y Takeda Science Foundation.

\section{Declaración de situación}

Los autores declaran que no poseen ningún conflicto de interés. 


\section{Referencias bibliográficas}

1 Varma R, Bressler NM, Doan QV, Gleeson M, Danese M, Bower JK, Selvin E, Dolan C, Fine J, Colman S, Turpcu A: Prevalence of and risk factors for diabetic macular edema in the United States. JAMA Ophthalmol 2014;132: 1334-1340.

2 Aiello LP, Avery RL, Arrigg PG, Keyt BA, Jampel HD, Shah ST, Pasquale LR, Thieme H, Iwamoto MA, Park JE, et al: Vascular endothelial growth factor in ocular fluid of patients with diabetic retinopathy and other retinal disorders. N Engl J Med 1994;331:1480-1487.

3 Antonetti DA, Barber AJ, Hollinger LA, Wolpert EB, Gardner TW: Vascular endothelial growth factor induces rapid phosphorylation of tight junction proteins occludin and zonula occluden 1. A potential mechanism for vascular permeability in diabetic retinopathy and tumors. J Biol Chem 1999;274:23463-23467.

4 Rajendram R, Fraser-Bell S, Kaines A, Michaelides M, Hamilton RD, Esposti SD, Peto T, Egan C, Bunce C, Leslie RD, Hykin PG: A 2-year prospective randomized controlled trial of intravitreal bevacizumab or laser therapy (BOLT) in the management of diabetic macular edema: 24-month data: report 3. Arch Ophthalmol 2012;130:972-979.

5 Elman MJ, Bressler NM, Qin H, Beck RW, Ferris FL 3rd, Friedman SM, Glassman AR, Scott IU, Stockdale CR, Sun JK; Diabetic Retinopathy Clinical Research Network: Expanded 2-year follow-up of ranibizumab plus prompt or deferred laser or triamcinolone plus prompt laser for diabetic macular edema. Ophthalmology 2011;118:609-614.

6 Nguyen QD, Brown DM, Marcus DM, Boyer DS, Patel S, Feiner L, Gibson A, Sy J, Rundle AC, Hopkins JJ, Rubio RG, Ehrlich JS; RISE and RIDE Research Group: Ranibizumab for diabetic macular edema: results from 2 phase III randomized trials: RISE and RIDE. Ophthalmology 2012;119:789-801.

7 Lang GE, Berta A, Eldem BM, Simader C, Sharp D, Holz FG, Sutter F, Gerstner O, Mitchell P; RESTORE Extension Study Group: Two-year safety and efficacy of ranibizumab $0.5 \mathrm{mg}$ in diabetic macular edema: interim analysis of the RESTORE extension study. Ophthalmology 2013;120:2004-2012.

8 Diabetic Retinopathy Clinical Research Network, Elman MJ, Aiello LP, Beck RW, Bressler NM, Bressler SB, Edwards AR, Ferris FL 3rd, Friedman SM, Glassman AR, Miller KM, Scott IU, Stockdale CR, Sun JK: Randomized trial evaluating ranibizumab plus prompt or deferred laser or triamcinolone plus prompt laser for diabetic macular edema. Ophthalmology 2010;117:1064-1077.e35.
9 Nguyen QD, Shah SM, Khwaja AA, Channa R, Hatef E, Do DV, Boyer D, Heier JS, Abraham $P$, Thach AB, Lit ES, Foster BS, Kruger E, Dugel P, Chang T, Das A, Ciulla TA, Pollack JS, Lim JI, Eliott D, Campochiaro PA; READ2 Study Group: Two-year outcomes of the ranibizumab for edema of the macula in diabetes (READ-2) study. Ophthalmology 2010; 117:2146-2151.

10 Diabetic Retinopathy Clinical Research Network, Scott IU, Edwards AR, Beck RW, Bressler NM, Chan CK, Elman MJ, Friedman SM, Greven CM, Maturi RK, Pieramici DJ, Shami M, Singerman LJ, Stockdale CR: A phase II randomized clinical trial of intravitreal bevacizumab for diabetic macular edema. Ophthalmology 2007;114:1860-1867.

11 Mitchell P, Bandello F, Schmidt-Erfurth U, Lang GE, Massin P, Schlingemann RO, Sutter F, Simader C, Burian G, Gerstner O, Weichselberger A; RESTORE Study Group: The RESTORE study: ranibizumab monotherapy or combined with laser versus laser monotherapy for diabetic macular edema. Ophthalmology 2011;118:615-625.

12 Michaelides M, Kaines A, Hamilton RD, Fraser-Bell S, Rajendram R, Quhill F, Boos CJ, Xing W, Egan C, Peto T, Bunce C, Leslie RD, Hykin PG: A prospective randomized trial of intravitreal bevacizumab or laser therapy in the management of diabetic macular edema (BOLT study) 12-month data: report 2. Ophthalmology 2010;117:1078-1086.e2.

13 Arevalo JF, Sanchez JG, Fromow-Guerra J, Wu L, Berrocal MH, Farah ME, Cardillo J, Rodriguez FJ; Pan-American Collaborative Retina Study Group (PACORES): Comparison of two doses of primary intravitreal bevacizumab (Avastin) for diffuse diabetic macular edema: results from the Pan-American Collaborative Retina Study Group (PACORES) at 12-month follow-up. Graefes Arch Clin Exp Ophthalmol 2009;247:735743.

14 Do DV, Nguyen QD, Boyer D, Schmidt-Erfurth U, Brown DM, Vitti R, Berliner AJ, Gao B, Zeitz O, Ruckert R, Schmelter T, Sandbrink R, Heier JS; da Vinci Study Group: One-year outcomes of the da Vinci Study of VEGF Trap-Eye in eyes with diabetic macular edema. Ophthalmology 2012;119:16581665.

15 Niwa Y, Kakinoki M, Sawada T, Wang X, Ohji M: Ranibizumab and aflibercept: intraocular pharmacokinetics and their effects on aqueous VEGF level in vitrectomized and nonvitrectomized macaque eyes. Invest Ophthalmol Vis Sci 2015;56:6501-6505.

16 Kakinoki M, Sawada O, Sawada T, Saishin Y, Kawamura H, Ohji M: Effect of vitrectomy on aqueous VEGF concentration and pharmacokinetics of bevacizumab in macaque monkeys. Invest Ophthalmol Vis Sci 2012;53: 5877-5880.
17 Yoshida S, Nakama T, Ishikawa K, Arima M, Tachibana T, Nakao S, Sassa Y, Yasuda M, Enaida H, Oshima Y, Kono T, Ishibashi T: Antiangiogenic shift in vitreous after vitrectomy in patients with proliferative diabetic retinopathy. Invest Ophthalmol Vis Sci 2012;53:6997-7003.

18 Yoshida S, Kubo Y, Kobayashi Y, Zhou Y, Nakama T, Yamaguchi M, Tachibana T, Ishikawa K, Arita R, Nakao S, Sassa Y, Oshima Y, Kono T, Ishibashi T: Increased vitreous concentrations of MCP-1 and IL-6 after vitrectomy in patients with proliferative diabetic retinopathy: possible association with postoperative macular oedema. $\mathrm{Br} \mathrm{J}$ Ophthalmol 2015;99:960-966.

19 Yanyali A, Aytug B, Horozoglu F, Nohutcu AF: Bevacizumab (Avastin) for diabetic macular edema in previously vitrectomized eyes. Am J Ophthalmol 2007;144:124-126.

20 Okamoto Y, Okamoto F, Hiraoka T, Oshika T: Vision-related quality of life and visual function following intravitreal bevacizumab injection for persistent diabetic macular edema after vitrectomy. Am J Ophthalmol 2014;58:369-374.

21 Bressler SB, Melia M, Glassman AR, Almukhtar T, Jampol LM, Shami M, Berger BB, Bressler NM; Diabetic Retinopathy Clinical Research Network: Ranibizumab plus prompt or deferred laser for diabetic macular edema in eyes with vitrectomy before anti-vascular endothelial growth factor therapy. Retina 2015;35:2516-2528.

22 Hoeben A, Landuyt B, Highley MS, Wildiers $\mathrm{H}$, Van Oosterom AT, De Bruijn EA: Vascular endothelial growth factor and angiogenesis. Pharmacol Rev 2004;56:549-580.

23 Ferrara N, Gerber HP, LeCouter J: The biology of VEGF and its receptors. Nat Med 2003;9:669-676.

24 Ebihara N, Matsuda A, Nakamura S, Matsuda $\mathrm{H}$, Murakami A: Role of the IL- 6 classic- and trans-signaling pathways in corneal sterile inflammation and wound healing. Invest Ophthalmol Vis Sci 2011;52:8549-8557.

25 Wang H, Lafdil F, Kong X, Gao B: Signal transducer and activator of transcription 3 in liver diseases: a novel therapeutic target. Int J Biol Sci 2011;7:536-550.

26 Yamada M, Kim S, Egashira K, Takeya M, Ikeda T, Mimura O, Iwao H: Molecular mechanism and role of endothelial monocyte chemoattractant protein-1 induction by vascular endothelial growth factor. Arterioscler Thromb Vasc Biol 2003;23:1996-2001.

27 Bressler SB, Qin H, Beck RW, Chalam KV, Kim JE, Melia M, Wells JA 3rd; Diabetic Retinopathy Clinical Research Network: Factors associated with changes in visual acuity and central subfield thickness at 1 year after treatment for diabetic macular edema with ranibizumab. Arch Ophthalmol 2012;130:11531161.
Ranibizumab para el tratamiento del

EMD después de una vitrectomía
Ophthalmologica 2017;238(suppl 1):21-27 DOI: $10.1159 / 000477513$ 